\title{
Measure density and extendability of Sobolev functions
}

\section{Piotr Hajłasz, Pekka Koskela and Heli Tuominen}

\begin{abstract}
We study necessary and sufficient conditions for a domain to be a Sobolev extension domain in the setting of metric measure spaces. In particular, we prove that extension domains must satisfy a measure density condition.
\end{abstract}

\section{Introduction}

In this paper we consider extension domains for Sobolev spaces. Recall that, for a domain $\Omega \subset \mathbb{R}^{n}$, the Sobolev space $W^{1, p}(\Omega), 1 \leq p<\infty$, consists of the functions $u \in L^{p}(\Omega)$ whose all first order weak derivatives $D_{j} u$ belong to $L^{p}(\Omega)$. We then write

$$
\|u\|_{W^{1, p}(\Omega)}=\|u\|_{L^{p}(\Omega)}+\sum_{j=1}^{n}\left\|D_{j} u\right\|_{L^{p}(\Omega)} .
$$

We say that $\Omega$ is a $W^{1, p}$-extension domain if there exists a bounded linear extension operator $E: W^{1, p}(\Omega) \rightarrow W^{1, p}\left(\mathbb{R}^{n}\right)$. It is well known that every bounded Lipschitz domain is a $W^{1, p}$-extension domain, for all $p$. In 1981, Jones gave a far reaching generalization of this fact by showing that this also holds for each so-called $(\varepsilon, \delta)$-domain, see [14]. He also showed that in some cases this result is best possible: a finitely connected planar $W^{1,2}$-extension domain is necessarily $(\varepsilon, \delta)$. It is immediate from the definition of $(\varepsilon, \delta)$-domains that the estimate

$$
|\Omega \cap B(x, r)| \geq C|B(x, r)|
$$

holds for each $x \in \bar{\Omega}$ and all $0<r<\delta$ (and so also for all $0<r \leq 1$ ).

2000 Mathematics Subject Classification: $46 \mathrm{E} 35$.

Keywords: Sobolev extension, doubling measure. 
It was very recently shown in [10] that (1.1) holds for all $W^{m, p}$-extension domains and that it together with the integrability of a suitable Calderón maximal function of each function in $W^{m, p}(\Omega)$ characterizes the extension domains. In the case of $m=1$ the condition on the integrability of the Calderón maximal function can be replaced by a pointwise inequality and the result from [10] reads as follows.

Proposition 1 If $\Omega \subset \mathbb{R}^{n}$ is a $W^{1, p}$-extension domain, $1 \leq p<\infty$, then the measure density condition (1.1) holds for all balls $B(x, r)$ with $x \in \bar{\Omega}$ and $0<r \leq 1$. Moreover, for $1<p<\infty$, the measure density condition (1.1) together with $W^{1, p}(\Omega)=M^{1, p}(\Omega)$ characterizes $W^{1, p}$-extension domains.

Here $M^{1, p}(\Omega)$ consist of all functions $u \in L^{p}(\Omega)$ for which the pointwise estimate

$$
|u(x)-u(y)| \leq|x-y|(g(x)+g(y))
$$

holds for some function $0 \leq g \in L^{p}(\Omega)$ for all $x, y \in \Omega \backslash E$, where $E$ has measure zero.

Let us note that, in the Euclidean setting, $M^{1, p}\left(\mathbb{R}^{n}\right)$ coincides with $W^{1, p}\left(\mathbb{R}^{n}\right)$ for $p>1,[6]$. However, $M^{1,1}$ identifies with a Hardy-Sobolev space by a recent result of Koskela and Saksman [17]. Note also that there are domains that do not have the extension property but for which nevertheless $M^{1, p}(\Omega)=W^{1, p}(\Omega)$, see [22].

There have been generalizations of the Jones' extension theorem to settings more general than the Euclidean one. Nhieu [20,21] established an analog of this result on Heisenberg and Carnot groups. Chua [3, 4] proved corresponding extension results for weighted Sobolev spaces where the weight is either in $A_{p}$, or it admits a Poincaré inequality. Very recently, Jones' extension theorem was further generalized to a metric setting by Björn and Shanmugalingam [1]. One is then led to ask if Proposition 1 admits a more general version. This turns out to be the case. For the necessary definitions, see Section 2.

Theorem 2 Let $X$ be a $Q$-regular, complete metric measure space that supports a $(1, p)$-Poincaré inequality for some $1 \leq p<\infty$. If $\Omega \subset X$ is an $N^{1, p}$-extension domain, then there exists a constant $C>0$ such that

$$
\mu(\Omega \cap B(x, r)) \geq C \mu(B(x, r))
$$

for all balls $B(x, r)$ with $x \in \bar{\Omega}$ and $0<r \leq 1$.

Moreover, for $1<p<\infty$, (1.3) together with $N^{1, p}(\Omega)=M^{1, p}(\Omega)$ characterizes $N^{1, p}$-extension domains. 
The upper bound 1 for the radius $r$ is not essential, any finite upper bound would do as well. If the measure $\mu$ satisfies only the lower mass bound $\mu(B(x, r)) \geq C r^{Q}$ for all balls, then the proof of Theorem 2 gives the estimate $\mu(\Omega \cap B(x, r)) \geq C r^{Q}$.

Notice that Heisenberg and Carnot groups are Ahlfors $Q$-regular spaces for a suitable $Q$ and that they support a $(1, p)$-Poincaré inequality for all $p \geq 1$. Moreover, the Sobolev space $N^{1, p}$ referred to in Theorem 2 reduces in the group setting to the Sobolev space considered by Nhieu [20], [21] and to that studied by Chua in the settings of [3], [4]. Furthermore, the setting of [1] is the same as ours.

Our next result, which is an analog of the corresponding result in [10], shows that the existence of a bounded linear extension operator follows from the apparently weaker assumption that each function in the Sobolev class has some extension.

Theorem 3 Fix $1<p<\infty$. Let $X$ be a $Q$-regular, complete metric measure space that supports a $(1, p)$-Poincaré inequality. Then $\Omega \subset X$ is an $N^{1, p_{-}}$ extension domain if and only if each $u \in N^{1, p}(\Omega)$ is the restriction to $\Omega$ of some function in $N^{1, p}(X)$.

The following theorem shows that the extension property is invariant under bi-Lipschitz mappings.

Theorem 4 Let $X, Y$ be $Q$-regular, complete metric measure spaces that support $(1, p)$-Poincaré inequalities, $1<p<\infty$. If $\Omega \subset X$ an $N^{1, p}$-extension domain and $f: \Omega \rightarrow f(\Omega) \subset Y$ is bi-Lipschitz, then $f(\Omega)$ is an $N^{1, p}$ extension domain.

Proposition 1 and Theorem 2 deal with extensions for the spaces $W^{1, p}$ and $N^{1, p}$ and $M^{1, p}$ plays only a role of a reference space. It is now natural to inquire for a formulation that only refers to $M^{1, p}$. Our next result answers this question.

Theorem 5 Let $X$ be a $Q$-regular, geodesic metric measure space. A domain $\Omega \subset X$ is an $M^{1, p}$-extension domain, $1 \leq p<\infty$, if and only if (1.3) holds.

Theorem 5 is new even in the Euclidean setting. As we have already mentioned, the space $M^{1,1}$ should be regarded as a counterpart of a HardySobolev space [17].

Our last result in this introduction shows that the measure density condition given in (1.3) guarantees the extendability of functions in the class $M^{1, p}$ provided $X$ is doubling. For a weaker result, see [11]. For a closely related, independent result see [26]. 
Theorem 6 Let $X$ be a doubling metric measure space. If for a closed set $F \subset X$, there is a constant $C_{F}>0$ such that

$$
\mu(F \cap B(x, r)) \geq C_{F} \mu(B(x, r))
$$

for all $x \in F$, and for all $0<r \leq 1$, then there is a bounded linear extension operator of $M^{1, p}(F)$ into $M^{1, p}(X)$ for all $1 \leq p<\infty$.

Here we understand $M^{1, p}(F)$ as the Sobolev space $M^{1, p}$ on the metric space $F$ with the metric inherited from $X$ and the measure being the restriction of the measure $\mu$ to $F$.

In the case $p>1$, Theorem 6 was proved independently and by a different method by Shvartsman [26] (an earlier version of our paper including this result has been available for some time). In the case $p>1$, we use boundedness of the maximal function in $L^{p}$, and hence this method cannot be used when $p=1$. To overcome this difficulty, we use a Sobolev-Poincaré inequality from [7] which allows us to work with exponents less than 1.

The paper is organized as follows. In Section 2 we introduce the notation and the standard assumptions used in the paper. Section 3 contains lemmas. We prove the main theorems in Section 4.

\section{Notation and preliminaries}

\subsection{Basic assumptions}

Throughout the paper, $X$ is a metric measure space equipped with a metric $d$ and a Borel regular measure $\mu$. We assume that the measure $\mu$ is doubling, that is, there is a fixed constant $C_{\mu}>0$, a doubling constant of $\mu$, such that

$$
\mu(B(x, 2 r)) \leq C_{\mu} \mu(B(x, r))
$$

whenever $x \in X$ and $r>0$. Here $B(x, r)=\{y \in X$ : dist $(y, x)<r\}$ is the open ball of radius $r$ centered at $x$. If $0<t<\infty$ and $B=B(x, r)$ is a ball in $X$, then $t B=B(x, t r)$. We also assume that open sets have positive and bounded sets finite measure. Recall that the doubling condition of $\mu$ implies that there exists a constant $C>0$ such that the lower bound

$$
\frac{\mu(B)}{\mu\left(B_{0}\right)} \geq C\left(\frac{r}{r_{0}}\right)^{s}
$$

holds with $s=\log _{2} C_{\mu}$ for all balls $B_{0}=B\left(x_{0}, r_{0}\right)$ and $B=B(x, r)$ whenever $x \in B_{0}$ and $0<r \leq r_{0}$, (see [9, Lemma 14.6]). Inequality (2.1) may hold with a smaller exponent than $\log _{2} C_{\mu}$; we call the smallest such $s$ the doubling dimension of $\mu$. As a special case of doubling spaces we consider $Q$-regular 
spaces. The space $X$ is (Ahlfors) $Q$-regular, $Q \geq 1$, if there is a constant $C_{Q} \geq 1$ such that

$$
C_{Q}^{-1} r^{Q} \leq \mu(B(x, r)) \leq C_{Q} r^{Q}
$$

for each $x \in X$, and for all $0<r \leq \operatorname{diam}(X)$. Here diam $(X)$ is the diameter of $X$.

A space $X$ is a geodesic space if every two points $x, y \in X$ can be joined by a curve whose length equals $d(x, y)$.

The Hardy-Littlewood maximal function of a function $u \in L_{\mathrm{loc}}^{1}(X)$ is

$$
\mathcal{M} u(x)=\sup _{0<r<\infty} f_{B(x, r)}|u| d \mu .
$$

Above, $u_{B}=f_{B} u d \mu=\mu(B)^{-1} \int_{B} u d \mu$ is the integral average of $u$ over the ball $B$. The local space $L_{\text {loc }}^{1}(X)$ consists of functions that are integrable in each ball.

By $\chi_{E}$, we denote the characteristic function of a set $E \subset X$, and by $|E|$, the Lebesgue $n$-measure of a measurable set $E \subset \mathbb{R}^{n}$. In general, $C$ will denote a positive constant whose value is not necessarily the same at each occurrence. If there is a positive constant $C_{1}$ such that $C_{1}^{-1} u \leq v \leq C_{1} u$, we write $u \approx v$, and say that $u$ and $v$ are comparable.

\subsection{Sobolev spaces $M^{1, p}(X)$ and $N^{1, p}(X)$}

Following [13], (cf. [16]), we say that a Borel measurable function $g \geq 0$ is an upper gradient of a function $u$ in an open set $\Omega \subset X$, if

$$
|u(x)-u(y)| \leq \int_{\gamma} g d s
$$

for each pair of points $x, y$ in $\Omega$, and all rectifiable curves $\gamma$ joining $x$ and $y$ in $\Omega$. Moreover, we say that $g$ is a $p$-weak upper gradient in $\Omega$ if (2.3) holds for $u$ and $g$ except for a family of compact, rectifiable curves in $\Omega$ with zero $p$-modulus. For the $p$-modulus on metric measure spaces and the properties of upper gradients, see for example [7], [13], [24], and [25].

The Sobolev space $N^{1, p}(\Omega)$, defined in [24], consists of those functions $u \in L^{p}(\Omega)$ that have a $p$-weak upper gradient $g \in L^{p}(\Omega)$ in $\Omega$. The space $N^{1, p}(\Omega)$ is a Banach space with the norm

$$
\|u\|_{N^{1, p}(\Omega)}=\|u\|_{L^{p}(\Omega)}+\inf \|g\|_{L^{p}(\Omega)},
$$

where the infimum is taken over all $p$-weak upper gradients, or, equivalently, over upper gradients $g \in L^{p}(\Omega)$ of $u$. Each $u \in N^{1, p}(X)$ has $a$ minimal $p$-weak upper gradient $g_{u} \in L^{p}(X)$, that is, $0 \leq g_{u} \leq g \mu$-almost everywhere in $X$ whenever $g \in L^{p}(X)$ is a $p$-weak upper gradient of $u$, see [25, Corollary 3.7], and [7, Theorem 7.16] for the case $p=1$. 
The Sobolev space $M^{1, p}(\Omega)$ was introduced via the pointwise inequality (1.2) in [6] (cf. [7]). We will need to use this space for other spaces than $X$ as well, so we provide a general definition. Let $\Omega$ be an open subset of a metric space $(Y, \rho)$, where the metric space $Y$ is equipped with a Borel regular measure $\nu$. For a measurable function $u$ in $\Omega$, we denote by $D(u)$ the class of all nonnegative measurable functions $g$ in $\Omega$ such that

$$
|u(x)-u(y)| \leq \rho(x, y)(g(x)+g(y)) \quad \nu \text {-a.e. }
$$

We call the elements of $D(u)$ generalized gradients of $u$. For $p>0$, the space $M^{1, p}(\Omega)=M^{1, p}(\Omega, \rho, \nu)$ consists of all $u \in L^{p}(\Omega)$ such that $D(u) \cap L^{p}(\Omega) \neq \emptyset$. For $u \in M^{1, p}(\Omega)$, we define

$$
\|u\|_{M^{1, p}(\Omega)}=\|u\|_{L^{p}(\Omega)}+\inf \|g\|_{L^{p}(\Omega)},
$$

where the infimum is taken over all functions $g \in L^{p}(\Omega) \cap D(u)$. Clearly (2.5) is a norm for $p \geq 1$ only, but we will need to consider this space for all $p>0$.

The space $M^{1, p}(X)$ always imbeds in $N^{1, p}(X)$. Indeed, if $u \in M^{1, p}(X)$ and $g \in D(u) \cap L^{p}(X)$, then the function $2 g$ is a $p$-weak upper gradient of $u$. The space $N^{1, p}(X)$ imbeds in $M^{1, p}(X)$ if $X$ supports a $(1, q)$-Poincaré inequality for some $1<q<p$, see [24, Theorems 4.8, 4.9], and [7, Theorems 8.6, 11.3]. If $X$ is complete, doubling and supports a $(1, p)$-Poincaré inequality, $1<p<\infty$, then $X$ supports a $(1, q)$-Poincaré inequality for some $1<q<p$ by a recent result of Keith and Zhong [15]. Hence for such spaces, $N^{1, p}(X)=M^{1, p}(X)$, and the norms are equivalent.

A pair of $u \in L_{\text {loc }}^{1}(\Omega)$ and a measurable function $g \geq 0$ satisfy a $(1, p)$ Poincaré inequality in $\Omega, p>0$, if there exist constants $C_{p}>0$ and $\tau \geq 1$ such that

$$
f_{B}\left|u-u_{B}\right| d \mu \leq C_{p} r\left(f_{\tau B} g^{p} d \mu\right)^{1 / p}
$$

for each ball $B=B(x, r)$ satisfying $\tau B \subset \Omega$. If there are constants $C_{p}>0$ and $\tau \geq 1$ such that the inequality (2.6) holds for each ball $B$ satisfying $\tau B \subset \Omega$, each function $u \in L_{\text {loc }}^{1}(B)$ and every upper gradient $g$ of $u$ in $\tau B$, then we say that $\Omega$ supports a $(1, p)$-Poincaré inequality.

Each pair $u \in M^{1, p}(X), g \in D(u)$, satisfies a $(1, q)$-Poincaré inequality for all $q \geq 1$; this follows by integrating inequality (1.2) twice and using the Hölder inequality, see [6, Lemma 2]. For the case $p \leq 1$, see Section 3 and [7].

Extension property for spaces $N^{1, p}$ and $M^{1, p}$ and for closed set are defined similarly as for $W^{1, p}$. For example, a domain $\Omega \subset X$ is an $N^{1, p}$-extension domain, if there is a bounded (linear) operator $E: N^{1, p}(\Omega) \rightarrow N^{1, p}(X)$ such that $\left.E u\right|_{\Omega}=u$ for all $u \in N^{1, p}(\Omega)$. 


\section{Lemmas}

Starting from the work of Jones [14], a Whitney type covering of the complement of the domain has been an essential tool when showing that the domain has an extension property. We begin this section by recalling a covering lemma for doubling metric measure spaces, [5, Theorem III.1.3], [18, Lemma 2.9]. Lemma 7 is usually used with the assumption that $U$ is bounded. However, this assumption is not necessary.

Let $U \subset X$ be open and $U \neq X$. For $x \in U$, let $r(x)=\operatorname{dist}(x, X \backslash U) / 10$. From the family of balls $\{B(x, r(x) / 5)\}_{x \in U}$ we select a maximal (countable) subfamily $\left\{B\left(x_{i}, r\left(x_{i}\right) / 5\right)\right\}_{i \in I}$ of pairwise disjoint balls and denote $\mathcal{B}=\left\{B_{i}\right\}_{i \in I}$, where $B_{i}=B\left(x_{i}, r_{i}\right), r_{i}=r\left(x_{i}\right)$. We will refer to the family $\mathcal{B}$ as the Whitney covering of $U$. The following lemma collects basic properties of the Whitney covering. These easily follow from the definition of $\mathcal{B}$; the last property is an easy consequence of the doubling property of the measure $\mu$.

Lemma 7 There is $M \in \mathbb{N}$ such that

1. the balls $B\left(x_{i}, r_{i} / 5\right)$ are pairwise disjoint,

2. $U=\bigcup_{i \in I} B\left(x_{i}, r_{i}\right)$,

3. $B\left(x_{i}, 5 r_{i}\right) \subset U$,

4. if $x \in B\left(x_{i}, 5 r_{i}\right)$, then $5 r_{i}<\operatorname{dist}(x, X \backslash U)<15 r_{i}$,

5. there is $x_{i}^{*} \in X \backslash U$ such that $d\left(x_{i}, x_{i}^{*}\right)<15 r_{i}$,

6. $\sum_{i=1}^{\infty} \chi_{B\left(x_{i}, 5 r_{i}\right)}(x) \leq M$ for all $x \in U$.

With such a Whitney covering of $U$ we can associate a Lipschitz partition of unity that we next describe. Let $\psi$ be a real smooth function with $\psi \equiv 1$ on $[0,1], \psi \equiv 0$ on $[3 / 2, \infty), 0 \leq \psi \leq 1$. Put

$$
\psi_{i}(x)=\psi\left(\frac{d\left(x, x_{i}\right)}{r_{i}}\right) .
$$

Then $\psi_{i} \equiv 1$ on $B\left(x_{i}, r_{i}\right)$, supp $\psi_{i} \subset B\left(x_{i}, 2 r_{i}\right)$ and the Lipschitz constant of $\psi_{i}$ is $C r_{i}^{-1}$. We define a partition of unity in a standard way by setting

$$
\varphi_{i}(x)=\frac{\psi_{i}(x)}{\sum_{j} \psi_{j}(x)} .
$$

The following properties of the functions $\varphi_{i}$ are easy to verify. 
Lemma 8 Let $\left\{B_{i}\right\}_{i \in I}$ be the Whitney covering of $U$ and $\left\{\varphi_{i}\right\}_{i \in I}$ the associated partition of unity. Then

1. $\operatorname{supp} \varphi_{i} \subset 2 B_{i}$,

2. $\varphi_{i}(x) \geq M^{-1}$ for all $x \in B_{i}$,

3. there is a constant $K$ such that each $\varphi_{i}$ is $K r_{i}^{-1}$-Lipschitz,

4. $\sum_{i=1}^{\infty} \varphi_{i}(x)=\chi_{U}(x)$.

We will frequently use a well known and easy to prove fact that if two Whitney balls $B_{i}, B_{j}$ are such that $5 B_{i} \cap 5 B_{j} \neq \emptyset$, then the radii of the balls are comparable and the points $x_{i}^{*}, x_{j}^{*}$ are not very far from each other.

The measure density condition (1.3) for an open set $\Omega$ says that the set cannot be too thin near the boundary. It also implies that the measure of the boundary of $\Omega$ is zero.

Lemma 9 If an open set $\Omega \subset X$ satisfies the measure density condition (1.3), then $\mu(\partial \Omega)=0$.

Proof. The measure density condition implies that no point of $\partial \Omega$ can be a point of density for $\partial \Omega$ and hence $\mu(\partial \Omega)=0$ by the Lebesgue differentiation theorem.

The following Leibniz rule for $M^{1, p}$ was proved in [8, Lemma 5.20].

Lemma 10 Let $u \in M^{1, p}(\Omega), \Omega \subset X$, and let $\varphi$ be a bounded L-Lipschitz function whose support is in $\Omega$. Then the function $u \varphi$ is in $M^{1, p}(X)$, and

$$
g=\left(g_{u}\|\varphi\|_{\infty}+L|u|\right) \chi_{\operatorname{supp} \varphi} \in D(u \varphi) \cap L^{p}(X)
$$

for all $g_{u} \in D(u) \cap L^{p}(\Omega)$.

Proof. The triangle inequality implies that

$$
|u(x) \varphi(x)-u(y) \varphi(y)| \leq L d(x, y)|u(x)|+|\varphi(y)||u(x)-u(y)|
$$

and

$$
|u(x) \varphi(x)-u(y) \varphi(y)| \leq L d(x, y)|u(y)|+|\varphi(x)||u(x)-u(y)| .
$$

Now it suffices to consider four easy cases depending on whether $x$ or $y$ belongs to $\operatorname{supp} \varphi$ or not, and the claim easily follows.

We close this section by recalling Sobolev inequalities from [9] and [7]. The first result of Lemma 11 is a weak-type inequality, and the second gives a local Hölder continuity estimate for $p$ strictly larger than the doubling dimension. Lemma 12 is a version of the Sobolev-Poincaré inequality for $M^{1, p}$-functions. As usual, $s$ is the doubling dimension of $\mu$ and $p^{*}=s p /(s-p)$ for $0<p<s$. 
Lemma 11 [9, Theorem 5.1] Let $u, g$ be a pair of functions satisfying the $(1, p)$-Poincaré inequality (2.6), and let $B \subset X$ be a ball of radius $r>0$.

1. If $p<s$, then

$$
\frac{\mu\left(\left\{x \in B:\left|u(x)-u_{B}\right|>t\right\}\right) t^{p^{*}}}{\mu(B)} \leq C r^{p^{*}}\left(f_{5 \tau B} g^{p} d \mu\right)^{p^{*} / p} .
$$

2. If $p>s$, then (a representative of) $u$ is locally Hölder continuous and

$$
|u(x)-u(y)| \leq C r^{s / p} d(x, y)^{1-s / p}\left(f_{5 \tau B} g^{p} d \mu\right)^{1 / p}
$$

for all $x, y \in B$.

In the lemma below, the measure $\nu$ need not be doubling.

Lemma 12 [7, Theorem 8.7] Let $(Y, \rho)$ be a metric space with a Borel measure $\nu$. Suppose that for all balls $B, B_{0}$ with radii $r, r_{0}$, the measure $\nu$ satisfies

$$
\frac{\nu(B)}{\nu\left(2 B_{0}\right)} \geq b\left(\frac{r}{r_{0}}\right)^{s} \quad \text { whenever } B \subset 2 B_{0} .
$$

If $u \in M^{1, p}\left(2 B_{0}\right), s /(s+1) \leq p<s$, and $g \in D(u)$ then

$$
\left(f_{B_{0}}\left|u-u_{B_{0}}\right|^{p^{*}} d \nu\right)^{1 / p^{*}} \leq C r_{0}\left(f_{2 B_{0}} g^{p} d \nu\right)^{1 / p}
$$

where the constant $C$ depends on $b, s$ and $p$ only.

\section{Proofs}

\subsection{Proof of Theorem 6}

Let $u \in M^{1, p}(F)$ and $g \in D(u) \cap L^{p}(F)$. We can assume that $F \neq X$; otherwise the claim is trivial. Although the function $g$ is defined on $F$ only, we identify it with a function defined on $X$ by assuming that $g$ equals zero in $X \backslash F$. Note that it follows from the measure density condition that $g_{B} \approx g_{B \cap F}$ for all balls $B$ centered in $F$ and having radius less than 1 . Note also that the measure density condition holds for balls of uniformly bounded radius because $\mu$ is doubling.

Let $\mathcal{B}=\left\{B_{i}\right\}_{i \in I}, B_{i}=B\left(x_{i}, r_{i}\right)$, be the Whitney covering of $X \backslash F$ and let $\left\{\varphi_{i}\right\}_{i \in I}$ be the associated Lipschitz partition of unity. Let $\mathcal{B}_{1}=\left\{B_{i}\right\}_{i \in J}$ be the collection of all balls from $\mathcal{B}$ with radius less than 1 . For each $i \in J$, let $x_{i}^{*}$ be as in Lemma 7 . We define

$$
B_{i}^{*}=B\left(x_{i}^{*}, r_{i}\right) .
$$


Observe that if $x \in 2 B_{i}, i \in J$, then

$$
B_{i}^{*} \subset B(x, 25 r(x)):=B_{x} .
$$

It follows from the measure density condition and the doubling property of $\mu$ that $\mu\left(B_{x}\right) \leq C \mu\left(B_{i}^{*} \cap F\right)$. Let

$$
V=\{x \in X: \operatorname{dist}(x, F)<8\} .
$$

For $x \in V \backslash F$, denote by $I_{x}$ the collection of all $i \in I$ such that $x \in 2 B_{i}$. Clearly the number of elements in $I_{x}$ is bounded by $M$ (Lemma 7 ). Note that if $i \in I \backslash J$, then $r_{i} \geq 1$ and hence dist $\left(2 B_{i}, F\right) \geq 8 r_{i} \geq 8$, so $2 B_{i} \cap V=\emptyset$, $i \notin I_{x}$. Accordingly, $I_{x} \subset J$ and therefore

$$
\sum_{i \in I_{x}} \varphi_{i}(x)=\sum_{i \in I} \varphi_{i}(x)=\sum_{i \in J} \varphi_{i}(x)=1 \quad \text { for } x \in V \backslash F .
$$

We define $\tilde{E} u$, the local extension of $u$, by

$$
\tilde{E} u(x)= \begin{cases}u(x), & \text { if } x \in F \\ \sum_{i \in J} \varphi_{i}(x) u_{B_{i}^{*} \cap F}, & \text { if } x \in X \backslash F .\end{cases}
$$

- Assume first that $p>1$. We will split the proof into several steps.

Claim 1: $\|\tilde{E} u\|_{L^{p}(X)} \leq C\|u\|_{L^{p}(F)}$. Let $x \in X \backslash F$. We have

$$
|\tilde{E} u(x)|=\left|\sum_{i \in J} \varphi_{i}(x) u_{B_{i}^{*} \cap F}\right| \leq \sum_{i \in I_{x}} f_{B_{i}^{*} \cap F}|u| d \mu \leq C f_{B_{x}}|u| d \mu \leq C \mathcal{M} u(x),
$$

and the claim follows from the Hardy-Littlewood maximal theorem (cf. [9, Theorem 14.13]). Although $u$ is defined in $F$ only, in the last two expressions of the above inequality we extend $u$ to $X \backslash F$ by zero, so $f_{B_{x}}|u| d \mu=$ $\mu\left(B_{x}\right)^{-1} \int_{B_{x} \cap F}|u| d \mu$.

Claim 2: $\tilde{E} u \in M^{1, p}(V)$. To this end it suffices to prove that

$$
|\tilde{E} u(x)-\tilde{E} u(y)| \leq C d(x, y)(\mathcal{M} g(x)+\mathcal{M} g(y)) \quad \text { for a.e. } x, y \in V .
$$

We have to consider four cases.

CAse 1: $x, y \in F$. We have

$$
\begin{aligned}
|\tilde{E} u(x)-\tilde{E} u(y)| & =|u(x)-u(y)| \leq d(x, y)(g(x)+g(y)) \\
& \leq d(x, y)(\mathcal{M} g(x)+\mathcal{M} g(y)) \quad \text { a.e. }
\end{aligned}
$$

because $g(x) \leq \mathcal{M} g(x)$ whenever $x$ is a Lebesgue point of $g$. 
CASE 2: $x \in V \backslash F, y \in F$. We have

$$
|\tilde{E} u(x)-\tilde{E} u(y)|=|\tilde{E} u(x)-u(y)| \leq\left|\tilde{E} u(x)-u_{B_{x} \cap F}\right|+\left|u_{B_{x} \cap F}-u(y)\right| .
$$

We will estimate the terms on the right hand side separately.

Since $\sum_{i \in I_{x}} \varphi_{i}(x)=1$ and $g \in D(u)$, we have

$$
\begin{aligned}
\left|\tilde{E} u(x)-u_{B_{x} \cap F}\right| & =\left|\sum_{i \in I_{x}} \varphi_{i}(x)\left(u_{B_{i}^{*} \cap F}-u_{B_{x} \cap F}\right)\right| \\
& \leq \sum_{i \in I_{x}} f_{B_{i}^{*} \cap F} f_{B_{x} \cap F}|u(w)-u(z)| d \mu(w) d \mu(z) \\
& \leq C f_{B_{x} \cap F} f_{B_{x} \cap F}|u(w)-u(z)| d \mu(w) d \mu(z) \\
& \leq C r(x) f_{B_{x}} f_{B_{x}}(g(w)+g(z)) d \mu(w) d \mu(z) \\
& \leq 2 C r(x) \mathcal{M} g(x) \leq C^{\prime} d(x, y) \mathcal{M} g(x)
\end{aligned}
$$

because $r(x) \leq \operatorname{dist}(x, F) \leq d(x, y)$. For the second term we have the estimate

$$
\begin{aligned}
\left|u_{B_{x} \cap F}-u(y)\right| & \leq f_{B_{x} \cap F}|u(z)-u(y)| d \mu(z) \\
& \leq f_{B_{x} \cap F} d(z, y)(g(z)+g(y)) d \mu(z) \\
& \leq C d(x, y)(g(y)+\mathcal{M} g(x)) .
\end{aligned}
$$

We used here the observation that $d(z, y) \leq C d(x, y)$ for $z \in B_{x} \cap F$. The above two estimates together imply the desired inequality.

CASE 3: $x, y \in V \backslash F$ and $d(x, y) \geq \min \{\operatorname{dist}(x, F)$, dist $(y, F)\}$. We may assume that $\operatorname{dist}(x, F) \leq \operatorname{dist}(y, F)$. We start with the estimate

$$
|\tilde{E} u(x)-\tilde{E} u(y)| \leq\left|\tilde{E} u(x)-u_{B_{x} \cap F}\right|+\left|u_{B_{x} \cap F}-u_{B_{y} \cap F}\right|+\left|\tilde{E} u(y)-u_{B_{y} \cap F}\right|
$$

and observe that $r(y)<d(x, y)$ and that $d(z, w) \leq C d(x, y)$ for all $z \in B_{x}$, $w \in B_{y}$. The rest is pretty much the same as in the Case 2. We leave details to the reader.

CASE 4: $x, y \in V \backslash F$ and $d(x, y)<\min \{\operatorname{dist}(x, F)$, dist $(y, F)\}$. We may assume that $\operatorname{dist}(x, F) \leq \operatorname{dist}(y, F)$. Since $\sum_{i \in I_{x} \cup I_{y}}\left(\varphi_{i}(x)-\varphi_{i}(y)\right)=0$, we 
have

$$
\begin{aligned}
|\tilde{E} u(x)-\tilde{E} u(y)| & =\left|\sum_{i \in I_{x} \cup I_{y}}\left(\varphi_{i}(x)-\varphi_{i}(y)\right)\left(u_{B_{i}^{*} \cap F}-u_{B_{x} \cap F}\right)\right| \\
& \leq C \sum_{i \in I_{x} \cup I_{y}} \frac{d(x, y)}{r(x)} f_{B_{i}^{*} \cap F} f_{B_{x} \cap F}|u(w)-u(z)| d \mu(w) d \mu(z) \\
& \leq C d(x, y) \mathcal{M} g(x) .
\end{aligned}
$$

In the last but one inequality we employed the fact that the functions $\varphi_{i}$, for $i \in I_{x} \cup I_{y}$ are Lipschitz continuous with the Lipschitz constant bounded by $\operatorname{Cr}(x)^{-1}$. Moreover, $B_{i}^{*} \subset B_{x}$ for each $i \in I_{x} \cup I_{y}$, and the proof of the last inequality follows from estimates very similar to those in (4.2).

Final step. The function $\tilde{E} u$ has good estimates in $V$, but we want estimates in $X$. To this end, we choose a cut-off function $\Psi: X \rightarrow[0,1]$ such that $\Psi \equiv 1$ in $F, \Psi \equiv 0$ in $X \backslash V$ and $\Psi$ is Lipschitz continuous with some universal Lipschitz constant $L$. Now we define an extension operator by

$$
E u=\Psi \tilde{E} u \text {. }
$$

It immediately follows from Lemma 10 that $E u \in M^{1, p}(X)$ and

$$
(C \mathcal{M} g+L|\tilde{E} u|) \chi_{V} \in D(E u) .
$$

Hence

$$
\|E u\|_{M^{1, p}(X)} \leq C\left(\|u\|_{L^{p}(F)}+\|g\|_{L^{p}(F)}\right) .
$$

The proof for the case $p>1$ is complete.

- CASE $p=1$. Note that if $R>0$ is any positive number, then there is $b>0$ (depending on $R$ ) such that the measure $\nu$ being the restriction of $\mu$ to $F$ satisfies

$$
\frac{\nu(B)}{\nu\left(2 B_{0}\right)} \geq b\left(\frac{r}{r_{0}}\right)^{s}
$$

whenever the balls $B, B_{0}$ of radii $r, r_{0}$ are centered at $F, B \subset 2 B_{0}$ and $r_{0}<R$. This inequality easily follows from (1.4) and (2.1).

Let $q=s /(s+1)$. If $r_{0}<R$, then $u \in M^{1,1}\left(2 B_{0}\right) \subset M^{1, q}\left(2 B_{0}\right), q^{*}=1$ and hence for $g \in D(u)$ Lemma 12 yields

$$
f_{B_{0}}\left|u-u_{B_{0}}\right| d \nu \leq C r_{0}\left(f_{2 B_{0}} g^{q} d \nu\right)^{1 / q}
$$

i.e.

$$
f_{B_{0} \cap F}\left|u-u_{B_{0} \cap F}\right| d \mu \leq C r_{0}\left(f_{2 B_{0}} g^{q} d \mu\right)^{1 / q} .
$$

Here, as before, we extend $g$ to $X \backslash F$ by zero. From now on, the measure $\nu$ will never appear. It was only used to derive the inequality (4.3). 
Claim 1: $|\tilde{E} u(x)-\tilde{E} u(y)| \leq C d(x, y)\left(\left(\mathcal{M} g^{q}\right)^{1 / q}(x)+\left(\mathcal{M} g^{q}\right)^{1 / q}(y)\right)$ for a.e. $x, y \in V$, provided $d(x, y)<1$.

Before we prove this inequality, let us note that $g^{q} \in L^{1 / q}, 1 / q>1$, and hence the Hardy-Littlewood maximal theorem yields $\left\|\left(\mathcal{M} g^{q}\right)^{1 / q}\right\|_{1} \leq C\|g\|_{1}$. Since the pointwise inequality is satisfied for points that are close to each other, the above inequality proves that $\tilde{E} u$ has a generalized gradient in $L^{1}$, on small balls in $V$. To obtain a global estimate, we will later need to localize $\tilde{E} u$ using a partition of unity and glue the estimates together.

Now let us return to the proof of the Claim 1 . As in the case $p>1$, we have to consider four cases.

CAse 1: $x, y \in F, d(x, y)<1$. We have

$|\tilde{E} u(x)-\tilde{E} u(y)| \leq d(x, y)(g(x)+g(y)) \leq d(x, y)\left(\left(\mathcal{M} g^{q}\right)^{1 / q}(x)+\left(\mathcal{M} g^{q}\right)^{1 / q}(y)\right)$

a.e. because $g(x) \leq\left(\mathcal{M} g^{q}\right)^{1 / q}(x)$ whenever $x$ is a Lebesgue point of $g^{q}$.

CASE 2: $x \in V \backslash F, y \in F, d(x, y)<1$. Then $r(x)=\operatorname{dist}(x, F) / 10<1 / 10$ and hence there is $x^{*} \in B_{x} \cap F$ such that $d\left(x, x^{*}\right)<15 r(x)$ and $d\left(x, x^{*}\right) \leq$ $d(x, y)<1$. Denote $B_{x^{*}}=B\left(x^{*}, 50 r(x)\right)$. It is easy to see that

$$
B_{x} \subset B_{x^{*}}, \quad 2 B_{x^{*}} \subset 5 B_{x} .
$$

We have

$$
|\tilde{E} u(x)-\tilde{E} u(y)| \leq\left|\tilde{E} u(x)-u_{B_{x} \cap \cap F}\right|+\left|u(y)-u_{B_{x} \cap F}\right| .
$$

We begin by estimating the first term as in (4.2):

$$
\begin{aligned}
& \left|\tilde{E} u(x)-u_{B_{x^{*} \cap F}}\right| \leq C f_{B_{x^{*} \cap F}} f_{B_{x^{*} \cap F}}|u(w)-u(z)| d \mu(w) d \mu(z) \\
& \leq 2 C f_{B_{x^{*} \cap F}}\left|u-u_{B_{x^{*}} \cap F}\right| d \mu \leq C^{\prime} r(x)\left(f_{2 B_{x^{*}}} g^{q} d \mu\right)^{1 / q} \\
& \leq C^{\prime \prime} r(x)\left(f_{5 B_{x}} g^{q} d \mu\right)^{1 / q} \leq C^{\prime \prime} d(x, y)\left(\mathcal{M} g^{q}\right)^{1 / q}(x) .
\end{aligned}
$$

To estimate the second term, we will use a telescoping argument as in the proof of Theorem 3.2 in [9]. Since we need to prove that the inequality holds almost everywhere, we may assume that $y$ is a Lebesgue point of $u$. Let $k$ be the least integer such that $B(y, d(x, y)) \subset 2^{k} B_{x^{*}}$. Then the radius of the ball $2^{k-1} B_{x^{*}}$ is at most $3 d(x, y)$ and hence

$$
2^{k+1} B_{x^{*}} \subset B(x, 13 d(x, y)) \subset B(x, 13) .
$$

We have

$$
\begin{aligned}
& \text { (4.5) }\left|u(y)-u_{B_{x^{*} \cap F}}\right| \leq\left|u(y)-u_{B(y, d(x, y)) \cap F}\right| \\
& +\left|u_{B(y, d(x, y)) \cap F}-u_{2^{k} B_{x^{*}} \cap F}\right|+\left|u_{2^{k} B_{x^{*}} \cap F}-u_{B_{x^{*}} \cap F}\right|=\alpha+\beta+\gamma .
\end{aligned}
$$


Now

$$
\begin{aligned}
\alpha & \leq \sum_{i=0}^{\infty}\left|u_{B\left(y, 2^{-i} d(x, y)\right) \cap F}-u_{B\left(y, 2^{-(i+1)} d(x, y)\right) \cap F}\right| \\
& \leq \sum_{i=0}^{\infty} f_{B\left(y, 2^{-(i+1)} d(x, y)\right) \cap F}\left|u-u_{B\left(y, 2^{-i} d(x, y)\right) \cap F}\right| d \mu \\
& \leq C \sum_{i=0}^{\infty} f_{B\left(y, 2^{-i} d(x, y)\right) \cap F}\left|u-u_{B\left(y, 2^{-i} d(x, y)\right) \cap F}\right| d \mu \\
& \leq C^{\prime} \sum_{i=0}^{\infty} 2^{-i} d(x, y)\left(f_{2 B\left(y, 2^{-i} d(x, y)\right)} g^{q} d \mu\right)^{1 / q} \\
& \leq 2 C^{\prime} d(x, y)\left(\mathcal{M} g^{q}\right)^{1 / q}(y) .
\end{aligned}
$$

The second term is estimated as follows:

$$
\begin{aligned}
\beta & \leq f_{B(y, d(x, y)) \cap F}\left|u-u_{2^{k} B_{x^{*} \cap F} \mid}\right| \mu \leq C f_{2^{k} B_{x^{*} \cap F}}\left|u-u_{2^{k} B_{x^{*} \cap F}}\right| d \mu \\
& \leq C^{\prime} d(x, y)\left(f_{2^{k+1} B_{x^{*} \cap F}} g^{q}\right)^{1 / q} \leq C^{\prime \prime} d(x, y)\left(\mathcal{M} g^{q}\right)^{1 / q}(x) .
\end{aligned}
$$

The estimate of the third part is pretty similar to that for the first part:

$$
\begin{aligned}
\gamma & \leq \sum_{i=0}^{k-1}\left|u_{2^{i} B_{x^{*}} \cap F}-u_{2^{i+1} B_{x^{*}} \cap F}\right| \\
& \leq \operatorname{Cr}(x) \sum_{i=0}^{k-1} 2^{i+1}\left(f_{2^{i+2} B_{x^{*}}} g^{q} d \mu\right)^{1 / q} \leq C^{\prime} d(x, y)\left(\mathcal{M} g^{q}\right)^{1 / q}(x) .
\end{aligned}
$$

In the last step, we used the fact that $2^{k} r(x) \approx d(x, y)$ and that each ball $2^{i+2} B_{x^{*}}$ is contained in a ball centered at $x$ of a comparable radius. The above estimates put together give the claim.

CASE 3: $x, y \in V \backslash F, d(x, y) \geq \min \{\operatorname{dist}(x, F)$, dist $(y, F)\}, d(x, y)<1$. We start with the inequality

$$
|\tilde{E} u(x)-\tilde{E} u(y)| \leq\left|\tilde{E} u(x)-u_{B_{x^{*}} \cap F}\right|+\left|\tilde{E} u(y)-u_{B_{y^{*}} \cap F}\right|+\left|u_{B_{x^{*}} \cap F}-u_{B_{y^{*}} \cap F}\right| .
$$

As in the corresponding case for $p>1$, we have that $r(x)<d(x, y)$ and $r(y)<d(x, y)$. Hence the estimates for the first two terms follow from (4.4). The estimate for the last term is similar to that of (4.5), but it involves more triangle inequalities. After tedious, but otherwise elementary computations one arrives at

$$
\left|u_{B_{x^{*} \cap F}}-u_{B_{y^{*} \cap F}}\right| \leq C d(x, y)\left(\left(\mathcal{M} g^{q}\right)^{1 / q}(x)+\left(\mathcal{M} g^{q}\right)^{1 / q}(y)\right) .
$$


CASE 4: $x, y \in V \backslash F, d(x, y)<\min \{\operatorname{dist}(x, F)$, dist $(y, F)\}, d(x, y)<1$. We may assume that dist $(x, F) \leq \operatorname{dist}(y, F)$. Arguments similar as in the case $p>1$ give

$$
\begin{aligned}
|\tilde{E} u(x)-\tilde{E} u(y)| & \leq C \frac{d(x, y)}{r(x)} f_{2 B_{x^{*} \cap F}} f_{2 B_{x^{*} \cap F}}|u(w)-u(z)| d \mu(w) d \mu(z) \\
& \leq C^{\prime} d(x, y)\left(f_{4 B_{x^{*}}} g^{q} d \mu\right)^{1 / q} \leq C^{\prime \prime} d(x, y)\left(\mathcal{M} g^{q}\right)^{1 / q}(x) .
\end{aligned}
$$

Final step. First we construct a partition of unity modifying arguments employed in Section 3. Let $\left\{\tilde{B}_{i}\right\}_{i=1}^{\infty}$ be a maximal family of pairwise disjoint balls of radius $1 / 10$ centered at $F$. Clearly

$$
U=\{x \in X: \operatorname{dist}(x, F)<1 / 10\} \subset \bigcup_{i=1}^{\infty} 3 \tilde{B}_{i}
$$

and it follows from the doubling condition of the measure that there is an integer $M \geq 1$ such that

$$
\sum_{i=1}^{\infty} \chi_{5 \tilde{B}_{i}}(x) \leq M \quad \text { for all } x \in X .
$$

Let $0 \leq \psi_{i} \leq 1$ be 5 -Lipschitz functions such that $\psi_{i}=1$ on $3 \tilde{B}_{i}$ and $\psi_{i}=0$ off $5 \tilde{B}_{i}$. Define

$$
\varphi_{i}(x)=\frac{\psi_{i}(x)}{\sum_{j=1}^{\infty} \psi_{j}(x)} .
$$

The functions $\varphi_{i}$ are $L$-Lipschitz in $U$ with $L$ depending on $M$ only. However, the functions $\varphi_{i}$ need not be well defined outside $U$, because the denominator may equal zero. Let $\zeta$ be a Lipschitz function on $X$ such that $\zeta \equiv 1$ on $F$ and $\zeta=0$ on $X \backslash U$. The functions $\eta_{i}=\zeta \varphi_{i}$ extend to Lipschitz functions on $X$ with a common bound for the Lipschitz constant, supp $\eta_{i} \subset 5 \tilde{B}_{i}$ and

$$
\Psi(x)=\sum_{i=1}^{\infty} \eta_{i}(x)=1 \quad \text { for } x \in F .
$$

The function $\Psi$ is Lipschitz continuous on $X$, because the supports of $\eta_{i}$ have bounded overlap. We will prove that

$$
E u=\Psi \tilde{E} u
$$

satisfies $\|E u\|_{M^{1,1}(X)} \leq c\|u\|_{M^{1,1}(F)}$ and hence $E u$ defines a bounded linear extension operator. 
For $x \in 5 \tilde{B}_{i}$ and $y \in F \cap 5 \tilde{B}_{i}$ we have $d(x, y)<1$ and hence the inequality from Claim 1 gives

$$
|\tilde{E} u(x)-u(y)| \leq C\left(\left(\mathcal{M} g^{q}\right)^{1 / q}(x)+\left(\mathcal{M} g^{q}\right)^{1 / q}(y)\right) .
$$

Integration over $x \in 5 \tilde{B}_{i}$ and $y \in F \cap 5 \tilde{B}_{i}$ yields

$$
f_{5 \tilde{B}_{i}}|\tilde{E} u| d \mu \leq C\left(f_{F \cap 5 \tilde{B}_{i}}|u| d \mu+f_{F \cap 5 \tilde{B}_{i}}\left(\mathcal{M} g^{q}\right)^{1 / q} d \mu+f_{5 \tilde{B}_{i}}\left(\mathcal{M} g^{q}\right)^{1 / q} d \mu\right) .
$$

Multiplication of this inequality by $\mu\left(5 \tilde{B}_{i}\right)$, and observation that $\mu\left(5 \tilde{B}_{i}\right) \approx$ $\mu\left(F \cap 5 \tilde{B}_{i}\right)$ gives

$$
\int_{5 \tilde{B}_{i}}|\tilde{E} u| d \mu \leq C\left(\int_{F \cap 5 \tilde{B}_{i}}|u| d \mu+\int_{5 \tilde{B}_{i}}\left(\mathcal{M} g^{q}\right)^{1 / q} d \mu\right) .
$$

The integrability of $\tilde{E} u$ over $5 \tilde{B}_{i}$, the fact that $d(x, y)<1$ for $x, y \in 5 \tilde{B}_{i}$ and the inequality from Claim 1 implies that $\tilde{E} u \in M^{1,1}\left(5 \tilde{B}_{i}\right)$. Therefore Lemma 10 implies that $\eta_{i} \tilde{E} u \in M^{1,1}(X)$ and

$$
g_{i}=C\left(\left(\mathcal{M} g^{q}\right)^{1 / q}+|\tilde{E} u|\right) \chi_{5 \tilde{B}_{i}} \in D\left(\eta_{i} \tilde{E} u\right)
$$

We have

$$
\begin{aligned}
\left\|\eta_{i} \tilde{E} u\right\|_{M^{1,1}(X)} & \leq \int_{5 \tilde{B}_{i}}|\tilde{E} u| d \mu+\int_{X} g_{i} d \mu \\
& \leq C\left(\int_{F \cap 5 \tilde{B}_{i}}|u| d \mu+\int_{5 \tilde{B}_{i}}\left(\mathcal{M} g^{q}\right)^{1 / q} d \mu\right) .
\end{aligned}
$$

Therefore the bounded overlap condition (4.6) and the Hardy-Littlewood theorem give

$$
\sum_{i=1}^{\infty}\left\|\eta_{i} \tilde{E} u\right\|_{M^{1,1}(X)} \leq C\|u\|_{M^{1,1}(F)} .
$$

Since $M^{1,1}(X)$ is a Banach space, [6], we conclude that

$$
E u=\sum_{i=1}^{\infty} \eta_{i} \tilde{E} u \in M^{1,1}(X) \quad \text { and } \quad\|E u\|_{M^{1,1}(X)} \leq C\|u\|_{M^{1,1}(F)} .
$$

The proof is complete. 


\subsection{Proof of Theorem 2}

If $\Omega$ is an $N^{1, p}$-extension domain, then the trace operator

$$
\mathcal{T}: N^{1, p}(X) \rightarrow N^{1, p}(\Omega), \quad \mathcal{T} v=\left.v\right|_{\Omega}
$$

is surjective. Hence the measure density condition (1.3) will follow from the following stronger result.

Proposition 13 Let $X$ be a $Q$-regular complete metric measure space that supports a $(1, p)$-Poincaré inequality for some $1 \leq p<\infty$. If $\Omega \subset X$ is a domain such that the trace operator (4.7) is surjective, then there is a constant $C>0$ such that

$$
\mu(\Omega \cap B(x, r)) \geq C \mu(B(x, r))
$$

for all balls $B(x, r)$ with $x \in \bar{\Omega}$ and $0<r \leq 1$.

Proof. Since $X$ supports the $(1, p)$-Poincaré inequality, $X$ is quasiconvex i.e., there is $C>0$ such that every two points $x, y \in X$ can be connected by a rectifiable curve $\gamma$ satisfying length $(\gamma) \leq C d(x, y)$ see $[9$, Proposition 4.4], [2]. This implies that $X$ endowed with the length metric $\rho(x, y)=\inf$ length $(\gamma)$, where the infimum is taken over all rectifiable curves connecting $x$ and $y$ is bi-Lipschitz homeomorphic to $X$. Since $Q$-regularity, $(1, p)$-Poincaré inequality and the $N^{1, p}(X)$ spaces are invariant under biLipschitz homeomorphisms (cf. [12, Chapter 9]), we may replace the metric $d$ by $\rho$ and work with the new space $(X, \rho, \mu)$. Therefore till the end of the proof we will assume that $d$ is the length metric i.e. it has the property that

$$
d(x, y)=\inf \operatorname{length}(\gamma)
$$

where the infimum is taken over all rectifiable curves connecting $x$ and $y$. Such a metric has an important property that every two points $x, y \in X$ can be connected by a geodesic i.e. a curve whose length equals $d(x, y)$, [7, Theorem 3.9].

Let $y \in \partial B(x, r)$ and $R<r$. Since $y$ can be connected to $x$ by a geodesic, it follows that the set $B(x, r) \cap B(y, R)$ contains a ball of radius $R / 2$, and hence

$$
\mu(B(y, R) \cap B(x, r)) \geq C \mu(B(y, R)) .
$$

This and the Lebesgue differentiation theorem implies that $\mu(\partial B(x, r))=0$, see Lemma 9.

The surjectivity of the trace operator implies that the space $N^{1, p}(\Omega)$ is isomorphic to $N^{1, p}(X) / \operatorname{ker} \mathcal{T}$ and hence there is $C>0$ such that for every $u \in N^{1, p}(\Omega)$ there is $v \in N^{1, p}(X)$ such that

$$
\|v\|_{N^{1, p}(X)} \leq C\|u\|_{N^{1, p}(\Omega)},\left.\quad v\right|_{\Omega}=u .
$$


This is to say that there is a bounded but not necessarily linear extension operator. Let $g_{E}$ be the minimal $p$-weak upper gradient of $v$.

CASE: $1 \leq p<Q$. For $0<r<1$, we choose $0<\tilde{\tilde{r}}<\tilde{r}<r$ such that

$$
\mu(B(x, \tilde{\tilde{r}}) \cap \Omega)=\frac{1}{2} \mu(B(x, \tilde{r}) \cap \Omega)=\frac{1}{4} \mu(B(x, r) \cap \Omega) .
$$

The existence of $\tilde{r}$ and $\tilde{\tilde{r}}$ follows from a fact that $\mu(\partial B(x, R))=0$ for every $R>0$. We denote

$$
A(\tilde{r}, \tilde{\tilde{r}})=B(x, \tilde{r}) \backslash B(x, \tilde{\tilde{r}}), \quad A(r, \tilde{r})=B(x, r) \backslash B(x, \tilde{r}),
$$

and define

$$
u(y)= \begin{cases}1, & \text { if } y \in B(x, \tilde{\tilde{r}}) \cap \Omega, \\ \frac{\tilde{r}-d(x, y)}{\tilde{r}-\tilde{r}}, & \text { if } y \in A(\tilde{r}, \tilde{\tilde{r}}) \cap \Omega, \\ 0, & \text { if } y \in \Omega \backslash B(x, \tilde{r}) .\end{cases}
$$

Clearly $u$ is Lipschitz with Lipschitz constant $1 /(\tilde{r}-\tilde{\tilde{r}})$. Hence the function

$$
g=\frac{1}{\tilde{r}-\tilde{\tilde{r}}} \chi_{\bar{A}(\tilde{r}, \tilde{\tilde{r}}) \cap \Omega}
$$

where $\bar{A}(\tilde{r}, \tilde{\tilde{r}})$ is the closure of the set $A(\tilde{r}, \tilde{\tilde{r}})$, is an upper gradient of $u$, (cf. [7, Lemma 6.7]). Hence

$$
\begin{aligned}
\|v\|_{N^{1, p}(X)} & \leq C\|u\|_{N^{1, p}(\Omega)} \\
& \leq C\left(\mu(B(x, \tilde{r}) \cap \Omega)^{1 / p}+(\tilde{r}-\tilde{\tilde{r}})^{-1} \mu(A(\tilde{r}, \tilde{\tilde{r}}) \cap \Omega)^{1 / p}\right) \\
& \leq C^{\prime}(\tilde{r}-\tilde{\tilde{r}})^{-1} \mu(B(x, \tilde{r}) \cap \Omega)^{1 / p} .
\end{aligned}
$$

Denote $B=B(x, r)$. To estimate the left hand side from below we will use the Sobolev inequality

$$
\left(\mu\left(\left\{x \in B:\left|v(x)-v_{B}\right|>t\right\}\right)\right)^{1 / p^{*}} \leq \frac{C}{t}\left(\int_{5 \tau B} g_{E}^{p} d \mu\right)^{1 / p} \leq \frac{C}{t}\|v\|_{N^{1, p}(X)}
$$

which is a direct consequence of Lemma 11 and the $Q$-regularity of the measure. Since $v=u=1$ on $B(x, \tilde{\tilde{r}}) \cap \Omega$ and $v=u=0$ on $A(r, \tilde{r}) \cap \Omega$, we conclude that

$$
\left|v-v_{B}\right| \geq \frac{1}{2}
$$

on at least one of the sets $B(x, \tilde{\tilde{r}}) \cap \Omega$ and $A(r, \tilde{r}) \cap \Omega$. The two sets have measures comparable to the measure of $B(x, \tilde{r}) \cap \Omega$ and hence the above inequality with $t=1 / 2$ yields

$$
\mu(B(x, \tilde{r}) \cap \Omega)^{1 / p^{*}} \leq 2 C\|v\|_{N^{1, p}(X)} \leq C^{\prime}(\tilde{r}-\tilde{\tilde{r}})^{-1} \mu(B(x, \tilde{r}) \cap \Omega)^{1 / p},
$$


and hence

$$
\tilde{r}-\tilde{\tilde{r}} \leq C \mu(B(x, \tilde{r}) \cap \Omega)^{1 / Q} \leq C \mu(B(x, r) \cap \Omega)^{1 / Q} .
$$

Now we define a sequence by setting

$$
r_{0}=r, \quad r_{j+1}=\tilde{r}_{j} .
$$

Clearly $\mu\left(B\left(x, r_{j}\right) \cap \Omega\right)=2^{-j} \mu(B(x, r) \cap \Omega)$ and hence $r_{j} \rightarrow 0$. The above inequality applied to $B\left(x, r_{j}\right)$ in the place of $B(x, r)$ gives

$$
r_{j+1}-r_{j+2} \leq C 2^{-j / Q} \mu(B(x, r) \cap \Omega)^{1 / Q} .
$$

This, in turn, gives

$$
\begin{aligned}
\tilde{r} & =\sum_{j=0}^{\infty}\left(r_{j+1}-r_{j+2}\right) \leq C\left(\sum_{j=0}^{\infty} 2^{-j / Q}\right) \mu(B(x, r) \cap \Omega)^{1 / Q} \\
& =C^{\prime} \mu(B(x, r) \cap \Omega)^{1 / Q},
\end{aligned}
$$

and the measure density condition easily follows from the following lemma, see [10, Lemma 13].

Lemma 14 If the measure density condition (4.8) holds for all $x \in \bar{\Omega}$ and all $0<r \leq 1$ such that $r \leq 10 \tilde{r}$, then it holds for all $x \in \bar{\Omega}$ and all $0<r \leq 1$.

Proof. Let $r \leq 1$. If $\Omega \subset B(x, r)$, then

$$
\mu(B(x, r) \cap \Omega)=\mu(\Omega) \geq \mu(\Omega) r^{Q} \geq C \mu(\Omega) \mu(B(x, r))
$$

and hence (4.8) is satisfied. If $r \leq 10 \tilde{r}$, then (4.8) is also satisfied. Thus we may assume that $\Omega \backslash B(x, r) \neq \emptyset$ and that $r>10 \tilde{r}$. Take $x^{\prime} \in B(x, r) \cap \Omega$ such that $d\left(x, x^{\prime}\right)=\tilde{r}+r / 5$. Such an $x^{\prime}$ exists because $\Omega \backslash B(x, r) \neq \emptyset$ and $\Omega$ is connected. Let $R=2 \tilde{r}+r / 5$. Then

$$
B(x, \tilde{r}) \subset B\left(x^{\prime}, R\right) \subset B(x, r)
$$

and

$$
B\left(x^{\prime}, R / 2\right) \subset B\left(x^{\prime}, r / 5\right) \subset A(r, \tilde{r}) .
$$

Hence $B(x, \tilde{r})$ and $B\left(x^{\prime}, R / 2\right)$ are disjoint subsets of $B\left(x^{\prime}, R\right)$ and thus

$$
\begin{aligned}
\mu\left(B\left(x^{\prime}, R / 2\right) \cap \Omega\right) & \leq \frac{1}{2}\left(\mu(A(r, \tilde{r}) \cap \Omega)+\mu\left(B\left(x^{\prime}, R / 2\right) \cap \Omega\right)\right) \\
& =\frac{1}{2}\left(\mu(B(x, \tilde{r}) \cap \Omega)+\mu\left(B\left(x^{\prime}, R / 2\right) \cap \Omega\right)\right) \\
& \leq \frac{1}{2} \mu\left(B\left(x^{\prime}, R\right) \cap \Omega\right) .
\end{aligned}
$$


This, in turn, implies that $\tilde{R} \geq R / 2$, and so the measure density condition is satisfied by the ball $B\left(x^{\prime}, R\right)$. Hence, using the $Q$-regularity, we have

$$
\begin{aligned}
\mu(B(x, r) \cap \Omega) & \geq \mu\left(B\left(x^{\prime}, R\right) \cap \Omega\right) \geq C \mu\left(B\left(x^{\prime}, R\right)\right) \\
& \geq C^{\prime} R^{Q} \geq C^{\prime \prime} \mu(B(x, r)) .
\end{aligned}
$$

The proof of the lemma is complete.

CASE: $p>Q$. Let $B=B(x, r)$ be a ball with $x \in \bar{\Omega}$ and $0<r \leq 1$. If $\Omega \subset B$, then condition (4.8) is satisfied. Thus we may assume that $\Omega \backslash B \neq \emptyset$. Let

$$
u(y)= \begin{cases}1-\frac{d(x, y)}{r} & \text { if } y \in B, \\ 0 & \text { if } y \in \Omega \backslash B,\end{cases}
$$

Clearly $u$ is $r^{-1}$-Lipschitz and the function

$$
g=\frac{1}{r} \chi_{\bar{B} \cap \Omega}
$$

is an upper gradient of $u$ (cf. [7, Lemma 6.7]). We have

$$
\begin{aligned}
\|v\|_{N^{1, p}(X)} & \leq C\|u\|_{N^{1, p}(\Omega)} \\
& \leq C\left(\mu(B(x, r) \cap \Omega)^{1 / p}+r^{-1} \mu(B(x, r) \cap \Omega)^{1 / p}\right) \\
& \leq 2 C^{\prime} r^{-1} \mu(B(x, r) \cap \Omega)^{1 / p} .
\end{aligned}
$$

Since $v(x)=u(x)=1$ and $v(y)=u(y)=0$ for some $y \in(\Omega \backslash B) \cap 2 B$, using the Sobolev inequality (3.1) and the $Q$-regularity, we conclude that

$$
\begin{aligned}
1 & \leq C r^{Q / p} r^{1-Q / p}\left(f_{10 \tau B} g_{E}^{p} d \mu\right)^{1 / p} \\
& \leq C^{\prime} r^{1-Q / p}\left(\int_{10 \tau B} g_{E}^{p} d \mu\right)^{1 / p} \\
& \leq C^{\prime} r^{1-Q / p}\|v\|_{N^{1, p}(X)} \\
& \leq C^{\prime \prime} r^{1-Q / p} r^{-1} \mu(B(x, r) \cap \Omega)^{1 / p},
\end{aligned}
$$

and inequality (4.8) easily follows.

CASE: $p=Q$. We will need the following result of Heinonen and Koskela [13, Theorem 5.9].

Lemma 15 Let $X$ be a Q-regular space that supports a (1,Q)-Poincaré inequality for some $Q \geq 1$. Let $E$ and $F$ be disjoint subsets of a ball $B=B(x, r)$ such that

$$
\min \left\{\mathcal{H}_{\infty}^{1}(E), \mathcal{H}_{\infty}^{1}(F)\right\} \geq \lambda r
$$

for some $0<\lambda \leq 1$. 
Then there is a constant $C \geq 1$, depending only on the data associated with $X$, such that

$$
\int_{10 \tau B} g^{Q} d \mu \geq C \lambda
$$

whenever $u \in L_{\mathrm{loc}}^{1}(X), g$ is an upper gradient of $u$ in $10 \tau B$, every $x \in E \cup F$ is a Lebesgue point of $u,\left.u\right|_{E} \geq 1$ and $\left.u\right|_{F} \leq 0$.

Recall that the Hausdorff 1 -content of a set $E$, used in Lemma 15, is the number $\mathcal{H}_{\infty}^{1}(E)=\inf \sum_{i} r_{i}$, where the infimum is taken over all countable covers of $E$ by balls of radius $r_{i}$. In [13], the sets $E$ and $F$ were assumed to be compact but this was not used in the proof. It was also assumed there that $u$ be continuous but this was only employed to guarantee that each $x \in E \cup F$ is a Lebesgue point of $u$.

Let $B=B(x, r)$ be a ball with $x \in \bar{\Omega}$ and $0<r \leq 1$. We may assume that $\Omega \backslash B(x, r) \neq \emptyset$ as otherwise (4.8) is satisfied. Let $A=\frac{2}{3} B \backslash \frac{1}{3} B$. The function

$$
u(y)= \begin{cases}1, & \text { if } y \in \frac{1}{3} B \cap \Omega, \\ 2-\frac{3 d(x, y)}{r}, & \text { if } y \in A \cap \Omega, \\ 0, & \text { if } y \in \Omega \backslash \frac{2}{3} B\end{cases}
$$

is $3 / r$-Lipschitz, and the function $g=3 r^{-1} \chi_{\bar{A} \cap \Omega}$ is an upper gradient of $u$ in $\Omega$. Similarly as in other cases we obtain the estimate

$$
\begin{aligned}
\|v\|_{N^{1, Q}(X)} & \leq C\|u\|_{N^{1, Q}(\Omega)} \leq C\left(\mu\left(\frac{2}{3} B \cap \Omega\right)^{1 / Q}+3 r^{-1} \mu(A \cap \Omega)^{1 / Q}\right) \\
& \leq C^{\prime} r^{-1} \mu(B \cap \Omega)^{1 / Q}
\end{aligned}
$$

We will estimate the left hand side of this inequality using Lemma 15. Let

$$
E=\frac{1}{3} B \cap \Omega, \quad F=\left(B \backslash \frac{2}{3} B\right) \cap \Omega .
$$

Observe first that

$$
\min \left\{\mathcal{H}_{\infty}^{1}(E), \mathcal{H}_{\infty}^{1}(F)\right\} \geq \frac{r}{3}
$$

Indeed, it follows from the connectivity of $\Omega$ that the 1-Lipschitz function $p$ : $\bar{\Omega} \rightarrow[0, \infty), p(y)=d(x, y)$ maps the sets $E$ and $F$ onto the intervals $[0, r / 3)$ and $[2 r / 3, r)$, respectively. As a 1-Lipschitz function does not increase the Hausdorff 1-content (easy exercise), we conclude that

$$
\mathcal{H}_{\infty}^{1}(E) \geq \mathcal{H}_{\infty}^{1}(p(E))=\frac{r}{3}, \quad \mathcal{H}_{\infty}^{1}(F) \geq \mathcal{H}_{\infty}^{1}(p(F))=\frac{r}{3}
$$


Hence Lemma 15 gives

$$
C \leq \int_{10 \tau B} g_{E}^{Q} d \mu \leq\|v\|_{N^{1, Q}(X)}^{Q} \leq C \frac{\mu(B \cap \Omega)}{r^{Q}}
$$

and the inequality (4.8) follows from the $Q$-regularity of the measure. This ends the proof of the proposition and hence that of the first part of the theorem.

Now we need to prove that $\Omega$ is an $N^{1, p}$-extension domain, $1<p<\infty$, if and only if $N^{1, p}(\Omega)=M^{1, p}(\Omega)$ and the condition (1.3) is satisfied.

Suppose that $\Omega$ is an $N^{1, p}$-extension domain and $u \in N^{1, p}(\Omega)$. Then (1.3) follows from the first part of the theorem. Since $X$ supports the $(1, p)$ Poincaré inequality, $N^{1, p}(X)=M^{1, p}(X)$ by [15]. Hence $E u$, the extension of $u$ belongs to $M^{1, p}(X)$, and so $u=\left.E u\right|_{\Omega} \in M^{1, p}(\Omega)$. Therefore $N^{1, p}(\Omega) \subset$ $M^{1, p}(\Omega)$, and thus $N^{1, p}(\Omega)=M^{1, p}(\Omega)$ because we always have $M^{1, p}(\Omega) \subset$ $N^{1, p}(\Omega)$ by [24], [7].

Suppose now that $N^{1, p}(\Omega)=M^{1, p}(\Omega)$ and the condition (1.3) is satisfied. Since $\mu(\partial \Omega)=0$ by Lemma 9 , we conclude that $M^{1, p}(\Omega)=M^{1, p}(\bar{\Omega})$. The claim follows from Theorem 6 and from the fact that $N^{1, p}(X)=M^{1, p}(X)$. The proof of the theorem is complete.

\subsection{Proof of Theorem 5}

The proof of the necessity part of Theorem 5 is a modification of the proof of Theorem 2. Since each pair $u \in M^{1, p}(B), g \in D(u)$, satisfies a $(1, q)$ Poincaré inequality for all $q \geq 1$, the assumption that $X$ supports a $(1, p)$ Poincaré inequality is not needed in this case.

Assume first that $\Omega$ is an $M^{1, p}$-extension domain. We present only the changes needed in the proof of Theorem 2 and leave it to the reader to check the details. In the case of $M^{1, p}(X)$, the norm minimizing generalized gradient exists only when $p>1$ (cf. [6, Theorem 2]). If $p=1$, instead of the minimal gradient, choose a generalized gradient $g_{E}$ of $v$ for which $\left\|g_{E}\right\|_{L^{1}(X)} \leq 2\|v\|_{M^{1,1}(X)}$.

In the case $1<p<Q$, notice first that $\mu(\partial B(x, r))=0$ for each ball $B(x, r)$ because $X$ is a geodesic space, see the beginning of the proof of Proposition 13. Use the generalized gradient $g=(\tilde{r}-\tilde{\tilde{r}})^{-1} \chi_{B(x, \tilde{r}) \cap \Omega}$ for $u$. Clearly $g \in D(u)$ because $u$ is $(\tilde{r}-\tilde{\tilde{r}})^{-1}$-Lipschitz and equals 0 outside $B(x, \tilde{r})$. The case $p>Q$ works using Lemma 11 . When $p=Q$, use the generalized gradient $g=3 r^{-1} \chi_{\frac{2}{3} B \cap \Omega}$ for $u$. Lemma 15 can be used for the pair $v, g_{E}$, because it satisfies a $(1, Q)$-Poincaré inequality.

The sufficiency part follows from Theorem 6 and Lemma 9. Namely, by the assumed measure density condition (1.3) and Lemma $9, \mu(\partial \Omega)=0$. 
By the definition of $M^{1, p}$, sets of zero measure are removable for $M^{1, p}$, and hence we have that $M^{1, p}(\Omega)=M^{1, p}(\bar{\Omega})$. Now we may use Theorem 6 for the set $F=\bar{\Omega}$. The proof is complete.

\subsection{Proof of Theorem 3}

The necessity part follows from the definition of an extension domain. Assume now that each function $u \in N^{1, p}(\Omega)$ is the restriction to $\Omega$ of some function in $N^{1, p}(X)$, that is, the trace operator (4.7) is surjective. Therefore Proposition 13 implies the measure density condition. By Theorem 2, it suffices to show that $N^{1, p}(\Omega)=M^{1, p}(\Omega)$. The proof of this fact is similar to the proof of the corresponding claim in Theorem 2 and is left to the the reader. The proof is complete.

\subsection{Proof of Theorem 4}

Let $f: \Omega \rightarrow f(\Omega)$ be a bi-Lipschitz mapping and suppose that $\Omega$ is an $N^{1, p}$-extension domain. By Theorem $2, \Omega$ satisfies the measure density condition (1.3) and $N^{1, p}(\Omega)=M^{1, p}(\Omega)$. Now $f(\Omega)$ satisfies (1.3) as biLipschitz homeomorphisms preserve the measure density condition. Moreover, the transformation $\Phi(u)=u \circ f$ induces isomorphisms of spaces, $\Phi: N^{1, p}(f(\Omega)) \rightarrow N^{1, p}(\Omega)$, and $\Phi: M^{1, p}(f(\Omega)) \rightarrow M^{1, p}(\Omega)$. Therefore $N^{1, p}(f(\Omega))=M^{1, p}(f(\Omega))$, and Theorem 2 shows that $f(\Omega) \subset Y$ is an $N^{1, p_{-}}$ extension domain.

Remark. Theorem 4 has an analog for $M^{1, p}, 1 \leq p<\infty$, by Theorem 5 . In this case the assumption of an $(1, p)$-Poincaré inequality is not needed.

\section{References}

[1] Björn, J. And Shanmugalingam, N.: Poincaré inequalities, uniform domains and extension properties for Newton-Sobolev functions in metric spaces. J. Math. Anal. Appl. 332 (2007), no. 1, 190-208.

[2] Cheeger, J.: Differentiability of Lipschitz functions on metric measure spaces. Geom. Funct. Anal. 9 (1999), 428-517.

[3] ChuA, S.-K.: Extension theorems on weighted Sobolev spaces. Indiana Univ. Math. J. 41 (1992), 1027-1076.

[4] ChuA, S.-K.: Some remarks on extension theorems for weighted Sobolev spaces. Illinois J. Math. 38 (1994), 95-126.

[5] Colfman, R. R. And Weiss G.: Analyse harmonique non-commutative sur certains espaces homogènes. Lecture Notes in Mathematics 242. Springer-Verlag, Berlin-New York, 1971. 
[6] Hajzasz, P.: Sobolev spaces on an arbitrary metric space. Potential Anal. 5 (1996), 403-415.

[7] HajŁasz, P.: Sobolev spaces on metric-measure spaces. In Heat kernels and analysis on manifolds, graphs, and metric spaces (Paris, 2002), 173-218. Contemp. Math. 338. Amer. Math. Soc. Providence, RI, 2003.

[8] HajŁasz, P. and Kinnunen, J.: Hölder quasicontinuity of Sobolev functions on metric spaces. Rev. Mat. Iberoamericana 14 (1998), no. 3, 601-622.

[9] HajŁasz, P. and Koskela, P.: Sobolev met Poincaré. Mem. Amer. Math. Soc. 145 (2000), no. 688.

[10] HajŁasz, P., Koskela, P. And Tuominen, T.: Sobolev embeddings, extensions and measure density condition. J. Funct. Anal. 254 (2008), no. 5, $1217-1234$.

[11] Harjulehto, P.: Sobolev extension domains on metric spaces of homogeneous type. Real Anal. Exchange 27 (2001/02), 583-597.

[12] Heinonen, J.: Lectures on analysis on metric spaces. Universitext. Springer-Verlag, New York, 2001.

[13] Heinonen, J. and Koskela, P.: Quasiconformal maps in metric spaces with controlled geometry. Acta Math. 181 (1998), 1-61.

[14] Jones, P. W.: Quasiconformal mappings and extendability of functions in Sobolev spaces. Acta Math. 147 (1981), 71-88.

[15] Keith, S. And Zhong, X.: The Poincaré inequality is an open ended condition. Ann. of Math. (2) 167 (2008), no. 2, 575-599.

[16] Koskela, P. And MacManus, P.: Quasiconformal mappings and Sobolev spaces. Studia Math. 131 (1998), no. 1, 1-17.

[17] Koskela, P. And Saksman, E.: Pointwise characterizations of HardySobolev functions. To appear in Math. Res. Lett.

[18] Macías, R. A. And Segovia, C.: A decomposition into atoms of distributions on spaces of homogeneous type. Adv. in Math. 33 (1979), 271-309.

[19] Mattila, P.: Geometry of sets and measures in Euclidean spaces. Cambridge Studies in Advanced Mathematics 44. Cambridge University Press, Cambridge, 1995.

[20] Nhieu, D.-M.: Extension of Sobolev spaces on the Heisenberg group. C. R. Acad. Sci. Paris Sér. I Math. 321 (1995), 1559-1564.

[21] Nhieu, D.-M.: The Neumann problem for sub-Laplacians on Carnot groups and the extension theorem for Sobolev spaces. Ann. Mat. Pura Appl. (4) 180 (2001), 1-25.

[22] Romanov, A.S.: On a generalization of Sobolev spaces. Siberian Math. J. 39 (1998), 821-824.

[23] Semmes, S.: Finding curves on general spaces through quantitative topology, with applications to Sobolev and Poincaré inequalities. Selecta Math. (N.S.) 2 (1996), 155-295. 
[24] Shanmugalingam, N.: Newtonian spaces: An extension of Sobolev spaces to metric measure spaces. Rev. Mat. Iberoamericana 16 (2000), 243-279.

[25] Shanmugalingam, N.: Harmonic functions on metric spaces. Illinois J. Math. 45 (2001), 1021-1050.

[26] Shvartsman, P.: On extensions of Sobolev functions defined on regular subsets of metric measure spaces. J. Approx. Theory 144 (2007), 139-161.

Recibido: 21 de mayo de 2007

Piotr Hajłasz

Department of Mathematics

University of Pittsburgh

301 Thackeray Hall, Pittsburgh, PA 15260, USA

hajlasz@pitt.edu

Pekka Koskela

Department of Mathematics and Statistics

P.O. Box 35 (MaD)

FI-40014 University of Jyväskylä, Finland

pkoskela@maths.jyu.fi

Heli Tuominen

Department of Mathematics and Statistics

P.O. Box $35(\mathrm{MaD})$

FI-40014 University of Jyväskylä, Finland

tuheli@maths.jyu.fi

Piotr Hajłasz was supported by the NSF grant DSM-0500966 and Pekka Koskela and Heli Tuominen by the Centre of Excellence Geometric Analysis and Mathematical Physics of the Academy of Finland. 